\title{
Modeling of Fuel Vapor Jet Eruption Induced by Local Droplet Heating
}

\author{
Jaeheon $\operatorname{Sim}^{1 *}$, Hong G. $\operatorname{Im}^{1,2 \dagger}$, Suk Ho Chung ${ }^{1 \ddagger}$ \\ ${ }^{1}$ King Abdullah University of Science and Technology, Thuwal 23955, Saudi Arabia \\ ${ }^{2}$ University of Michigan, Ann Arbor, MI 48109, USA
}

\begin{abstract}
The evaporation of a droplet by non-uniform heating is numerically investigated in order to understand the mechanism of the fuel-vapor jet eruption observed in the flame spread of a droplet array under microgravity condition. The phenomenon was believed to be mainly responsible for the enhanced flame spread rate through a droplet cloud at microgravity conditions. A modified Eulerian-Lagrangian method with a local phase change model is utilized to describe the interfacial dynamics between liquid droplet and surrounding air. It is found that the localized heating creates a temperature gradient along the droplet surface, induces the corresponding surface tension gradient, and thus develops an inner flow circulation commonly referred to as the Marangoni convection. Furthermore, the effect also produces a strong shear flow around the droplet surface, thereby pushing the fuel vapor toward the wake region of the droplet to form a vapor jet eruption. A parametric study clearly demonstrated that at realistic droplet combustion conditions the Marangoni effect is indeed responsible for the observed phenomena, in contrast to the results based on constant surface tension approximation.
\end{abstract}

\section{Nomenclature}

$c_{p} \quad$ specific heat

g gravitational acceleration vector

$h_{f g} \quad$ latent heat of the droplet

$k$ thermal conductivity

$\dot{m}_{f}$ mass evaporation rate

n unit normal vector

$p \quad$ hydrodynamic pressure

$T$ temperature

u velocity vector

$\kappa \quad$ local curvature of interface

$\mu \quad$ viscosity

$\rho \quad$ density

$\sigma \quad$ surface tension

Subscript

$l, g \quad$ liqud, gas

$f \quad$ interface between liquid and gas

\section{Introduction}

$\mathrm{U}$ 
systems, however, flames are spread through a spray cloud, and the physical process is much more complicated due to interactions between burning and unburned droplets that make the direct application of the single droplet analysis invalid.

As a baseline study of spray combustion, flame spreads along droplet arrays has been studied by Reichenbach et al, in which a correlation between the flame propagation times for various droplet sizes and spacings with the immersion depth. Brzustowski et al. ${ }^{6}$ measured flame transfer time of hydrocarbon droplets in free-fall experiments, and showed that ignition delay of the droplet is much longer than flame spread over a droplet, resulting in non-uniform velocity of flame propagation. It is known that, at normal gravity conditions, the flame spread rate decreases monotonically with pressure and ceases to propagate beyond a certain pressure. ${ }^{7}$ Under microgravity conditions, however, Kobayashi et al. ${ }^{8}$ showed a nonmonotonic response in that the flame spread rate reaches a minimum value at about half the critical pressure, increases to a maximum value at above critical pressure, and then decreases again. The fuel vapor jet from the evaporating droplet was observed by back-lit images, which contributed to increasing the flame spread rate around the critical pressure by enhancing heat and mass transfer to the unburned droplet. A similar jet-like flow was also observed in the flame spread over electrical wire. ${ }^{9}$ It was conjectured ${ }^{8}$ that the Marangoni effect induced by the temperature-dependent surface tension may be responsible for the fuel vapor jet eruption behavior, but it has not been clearly demonstrated or confirmed as of today.

Considering the difficulties in experimental measurements of detailed internal motion within the droplet, high fidelity computational model is suggested to investigate the physical mechanism of the unusual observations. This requires multiphase interfacial dynamics model considering detailed surface tension and phase change characteristics. To ensure the fidelity of the simulation, the interface location is must be tracked accurately, since the surface tension computation is directly related to the accuracy of interface geometry. Among many existing computational methods, ${ }^{10}$ an Eulerian-Lagrangian method (e.g., immersed boundary method) is selected in the present study in favor of its explicit representation and tracking of moving interface surface. ${ }^{11}$ The surface tension force on a discretized interface can be evaluated by direct curvature computation using surface fitting. However, interpolation-based curvature computation is numerically sensitive to data smoothing, and does not enforce the surface tension conservation of a droplet. The computation of line integral form using normal and tangent vector of the interface element is much easier, and maintains conservation without expensive curvature computation. ${ }^{12}$

For the phase change simulation, the amount of mass transfer must be computed accurately, and accurate determination of the latent heat is essential in obtaining correct interfacial condition. Juric and Tryggvason ${ }^{13}$ treated the latent heat as a source term of energy equation implicitly using a Newton iteration technique with the mass transfer rate treated as an unknown quantity. The method was simplified by Shin and Juric ${ }^{14}$ with explicit mass transfer computation while maintaining the diffused latent heat source term. Although this approach offers a simple solution, the accuracy tends to degrade at higher phase change rate. Alternatively, Son et al. ${ }^{15}$ solved the boiling bubbles problem using the level set method by assuming the temperature inside the bubble to be constant without the latent heat source term. The homogeneous temperature assumption was commonly accepted for its simplicity, and was adopted in the volume-of-fluids (VOF) method by Welch and Wilson. ${ }^{16}$ Morgan ${ }^{17}$ suggested another approach by a linear correction of the temperature within one cell on each side of the interface. Unfortunately, all of these approaches are not applicable to the present study in which consideration of the nonuniform temperature within the liquid phase is essential.

The present study attempts to develop an appropriate physical model to predict the fuel vapor jet from evaporating droplet. An Eulerian-Lagrangian method ${ }^{11,18}$ is implemented for the interfacial dynamics including surface tension along droplet surface and evaporation phenomena. The numerical algorithm utilizes the stationary Eulerian grids to describe the flow field, and moving Lagrangian surface meshes to treat the phase boundaries. Temperature-dependent surface tension model and the new local phase change model are employed. It is found that the level of temperature gradient encountered in the experimental conditions is indeed sufficient to produce a significant effect of the Marangoni convection and strong shear flow around the droplet, thereby leading the fuel vapor jet eruption. The finding was further confirmed by comparing the simulation results with a constant surface tension assumption.

\section{Numerical Method}

The phenomena of interest are essentially multi-dimensional and unsteady, and thus a typical 1-D quasi-steady droplet evaporation models are not adequate. In the present study, a marker-based 3-D Eulerian-Lagrangian method ${ }^{18}$ is implemented with a new local phase change model to perform the multiphase interfacial computations of an evaporating droplet heated by a localized energy source. 


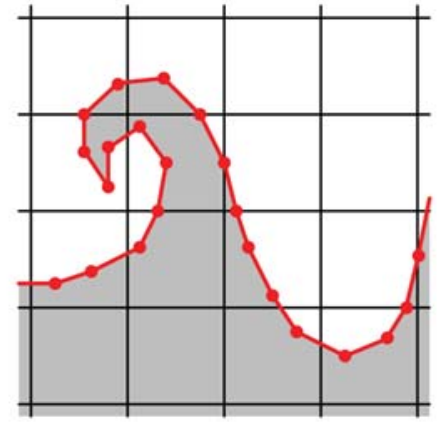

(a)

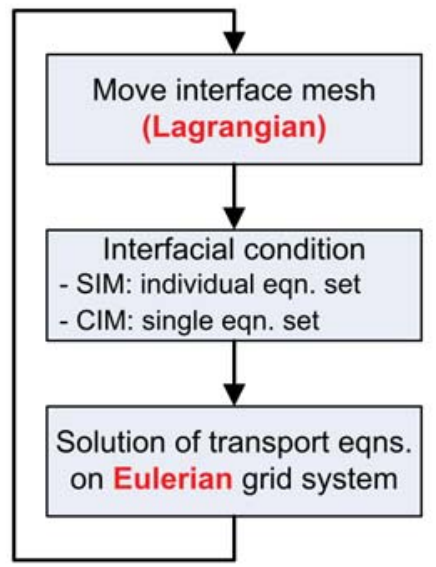

(b)

Figure 1: Illustration of the present Eulerian-Lagrangian methods. (a) Interface representation using moving (Lagrangian) meshes on the stationary (Eulerian) Cartesian grids. (b) Flowchart for the solution procedure. Both continuous interface method (CIM) with continuous forcing in a smeared interface zone and sharp interface method (SIM) with discrete forcing at a zero-thickness interface can be implemented. In the present research, CIM is implemented in general, and SIM is applied on the local phase change region.

\section{A. Multiphase modeling: Eulerian-Lagrangian method}

The multiphase flow including interfacial dynamics between liquid droplet and surrounding gas is simulated by the Eulerian-Lagrangian method ${ }^{18}$ in favor of its accurate interface representation and explicit tracking. Accurate interface geometry is very important since it determines the accuracy of surface tension computation.

The conservation equations for mass, momentum, and energy are written in Eqs. (1)-(3), which account for the interfacial dynamics as source terms in the governing equations; surface tension effects of fluid interfaces as a momentum forcing term $\mathbf{F}_{f}$; and the latent heat effects across fluid interfaces as a energy source term $Q_{f}$.

$$
\begin{gathered}
\frac{\partial \rho}{\partial t}+\nabla \cdot(\rho \mathbf{u})=0 \\
\frac{\partial(\rho \mathbf{u})}{\partial t}+\nabla \cdot(\rho \mathbf{u u})=-\nabla p+\nabla \cdot \mu\left(\nabla \mathbf{u}+\nabla^{\mathrm{T}} \mathbf{u}\right)+\mathbf{F}_{f}+\rho \mathbf{g} \\
\frac{\partial\left(\rho c_{p} T\right)}{\partial t}+\nabla \cdot\left(\rho c_{p} T \mathbf{u}\right)=\nabla \cdot(k \nabla T)+Q_{f}
\end{gathered}
$$

In general, there exist discontinuities across interface between different phases. Equations (4)-(6) shows these phase boundary conditions accounting for discontinuities in mass and heat transfer due to phase change, and pressure and viscous stress jump in the normal direction of interface.

$$
\begin{gathered}
\dot{m}_{f}=\rho_{1}\left(\mathbf{u}_{f}-\mathbf{u}_{1}\right) \cdot \mathbf{n}=\rho_{2}\left(\mathbf{u}_{f}-\mathbf{u}_{2}\right) \cdot \mathbf{n} \\
\left(p_{2}-p_{1}\right)-\mathbf{n} \cdot\left(\tau_{2}-\tau_{1}\right) \cdot \mathbf{n}=\sigma \kappa \\
\left(\mathbf{q}_{1}-\mathbf{q}_{2}\right) \cdot \mathbf{n}=\dot{m}_{f} h_{f g}
\end{gathered}
$$

In the present Eulerian-Lagrangian method, the governing equations of bulk flow variables are solved on the stationary (Eulerian) background grid with interfacial boundary conditions. The interfaces between different phases, liquid and gas in this study, are represented and tracked by moving (Lagrangian) surface meshes, which are established by line-segments in two-dimensional and triangles in three-dimensional domain. The Lagrangian meshes move freely on the Eulerian grid by the velocities at its location determined by:

$$
\frac{\partial \mathbf{x}_{f}}{\partial t}=\mathbf{u}_{f}\left(\mathbf{x}_{f}\right)=\mathbf{V}_{\text {fluid movement }}+\mathbf{V}_{\text {phase change }}
$$

The overall illustration of the present Eulerian-Lagrangian method and computational process is shown in figure 1. 


\section{B. Interfacial dynamics: continuous interface method (CIM)}

In the present study, the interfacial dynamics with discontinuity and surface tension between gas and liquid is modeled by the continuous interface method, where the material properties across interface are smoothed out by an approximate Dirac-delta function $\left(\delta_{h}\right)^{19}$ within 4 cell widths, such that a single set of equations for all fluid phases is solved in the entire domain. The smoothed fluid properties across interface are computed by:

$$
\begin{gathered}
\phi=\phi_{2}+\left(\phi_{1}-\phi_{2}\right) \mathrm{I}(\mathbf{x}) \\
\mathrm{I}(\mathbf{x})=\mathrm{H}\left(r=\mathbf{n} \cdot\left(\mathbf{x}-\mathbf{x}_{f}\right)\right)=\int_{-\infty}^{r} \delta_{h}(h) \mathrm{d} h
\end{gathered}
$$

where the indicator function (I) is a discrete form of the Heaviside step function in Eq. (9). Here, $\phi$ can be $\rho, \rho / \mu$, $\rho / c_{p}$ and $\rho c_{p} / k$.

The surface tension force of interface between liquid and gas is incorporated into the Eulerian grids as a function of the surface tension $(\sigma)$ at a local interface temperature $\left(T_{f}\right)$ and the local curvature of interface $(\kappa)$, given by:

$$
\mathbf{F}_{f}=\int_{\Gamma(t)} \sigma\left(T_{f}\right) \kappa \delta_{h}\left(\mathbf{x}-\mathbf{x}_{f}\right) d \Gamma
$$

Surface tension is a given material property, which typically increases with temperature. In this study, a piecewise linear approximation is used to compute accurate surface tension according to the temperature at each location along interface. The line integral form is used with normal and tangential direction of interface elements instead of direct curvature computation. This continuous interface method can be effective in modeling even highly deformable and moving droplet surfaces.

\section{Phase change modeling for evaporation}

During evaporation process, the liquid is transferred to the fuel vapor, and the difference of enthalpy is used for evaporation energy. Therefore, the critical modeling issues are (1) how to compute the mass transfer and (2) how to treat the latent heat, since they are directly related to the vapor concentration, phase boundaries movement, and the resulting temperature distribution.

In the present study, the mass transfer across interface is computed from interfacial boundary condition based on the Stefan condition given by:

$$
\dot{m}_{f}=\frac{\left(\mathbf{q}_{1}-\mathbf{q}_{2}\right) \cdot \mathbf{n}}{h_{f g}}=\frac{1}{h_{f g}}\left[-\left.k_{1} \frac{\partial T}{\partial \mathbf{n}}\right|_{1}+\left.k_{2} \frac{\partial T}{\partial \mathbf{n}}\right|_{2}\right]
$$

in which the heat flux difference is determined by the temperature gradient in each phase. The discontinuous thermal conductivity, $k_{1}$ and $k_{2}$, are utilized for accurate mass transfer computation even though the overall energy equation is solved with smoothed material properties via aforementioned continuous interface method.

When the mass transfer is determined, it should be reflected in the mass conservation equation of Eq. (4) during the computation. In the incompressible flow computation, this equation is simplified to divergence-free condition $(\nabla \cdot \mathbf{u}=0)$, and is the basis of the implemented incompressible projection method. With a phase change effect, however, the divergence of the velocity is not zero around the interface due to mass transfer across interface. In the present approach, the divergence of velocity is computed by using the Rankine-Hugoniot jump condition, Eq. (4), and mass transfer, Eq. (11), yielding

$$
\nabla \cdot \mathbf{u}=\left(\mathbf{u}_{1}-\mathbf{u}_{2}\right) \cdot \mathbf{n}=\dot{m}_{f}\left(\frac{1}{\rho_{1}}-\frac{1}{\rho_{2}}\right)
$$

In the present study, only one set of the governing equations including energy equation is solved throughout the entire domain using the continuous interface method. However, the phase change occurs when the local interface temperature reaches the boiling temperature. For simplicity, the boiling temperature is assumed equal to the saturation temperature at a given ambient pressure, which is an adequate assumption in macroscopic problems. For the latent heat implementation and evaporation computation, the energy equation is solved with a locally patched interface boundary condition by the ghost cell methodology, which is applied only in the region where phase change occurs, as shown in Figure 2. 


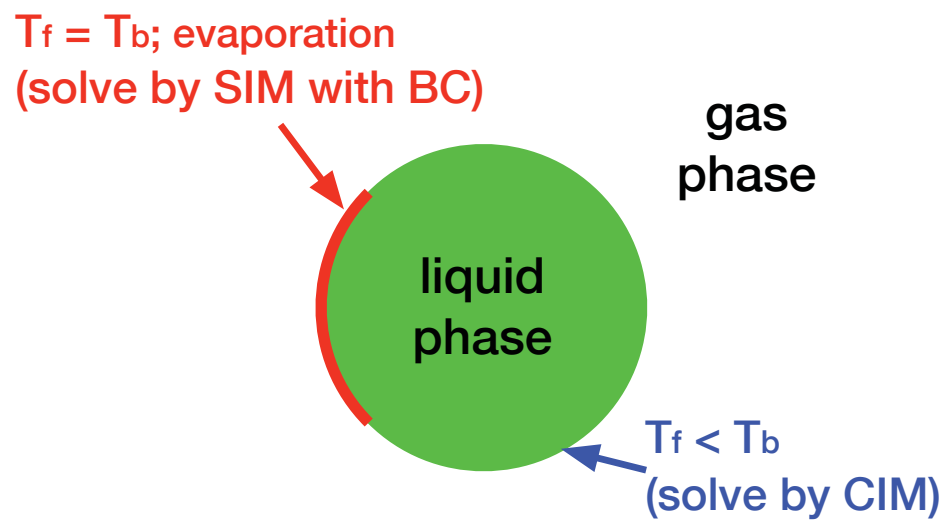

Figure 2: The illustration of the present local phase change model. Phase change (e.g., evaporation) computation is applied locally by the sharp interface method (SIM) with explicit interfacial boundary condition on the region where local interface temperature $\left(T_{f}\right)$ reaches the boiling temperature $\left(T_{b}\right)$. In other regions, continuous interface method (CIM) is implemented with smoothed material properties.

\section{Results and Discussion}

\section{A. Validation of the present evaporation model: Stefan problems}

As a validation of the developed method, a one dimensional two-phase Stefan problem is considered for comparison with exact solutions. Figure 3 shows the configuration of the two-phase flow on a semi-infinite slab, where the left wall was maintained at a constant temperature $T_{0}$ with the interface location, $x(t)$, separating the vapor and liquid phases on the left and right sides, respectively. The initial temperature of phase 2 is at $T_{\infty}$, which varies in time due to heat transfer from phase 1 .

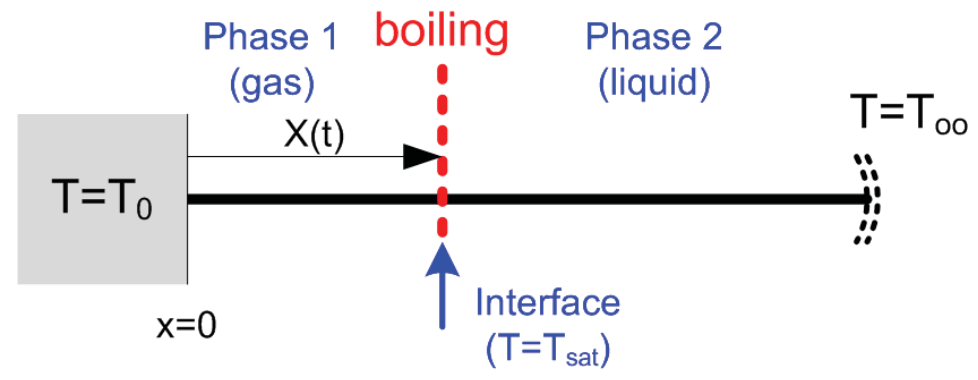

Figure 3: The numerical configuration of one-dimensional two-phase Stefan problem.

A low Jacob number $\left(\mathrm{Ja}=\rho_{l} c_{p} \Delta T / \rho_{v} h_{f g}\right)$ case is first validated with different thermal conductivity ratio of 0.1 to 10. The computations are started with the initial interface location at $x(t)=0.1$, assuming $T_{0}=1$ at the left end and $T_{\infty}=-1$ at the right end with the saturation temperature $T_{\text {sat }}=0$. The theoretical temperature distribution at the time that corresponds to the initial interface location is imposed as the initial condition of the present numerical study. The Jacob number is 2.0 in these cases. The interface location history during phase change process is shown in figure 4(a) for the different thermal conductivity ratio of $0.1,1$ and 10. The interface moves fast initially and slows down as the temperature gradient becomes smaller due to the interface moving away from the hot temperature at $x=0$. The results at a low Jacob number in figure 4(a) show good agreement with the analytical solutions. Higher Jacob number cases are also validated for faster evaporation or higher density ratio. In the droplet evaporation case under study, the Jacob number is estimated to be approximately 200 at $1 \mathrm{MPa}$, and increases rapidly with pressure. The small latent heat $\left(h_{f g}\right)$ at higher pressure causes larger amount of mass transfer with the same amount of heat transfer, and the droplet shrinks very rapidly due to the high evaporation rate. These are more complex and computationally demanding problems. Figure 4(b) shows that the present new phase change model works very well even at a very high Jacob number of $\mathrm{Ja}=1000$. 


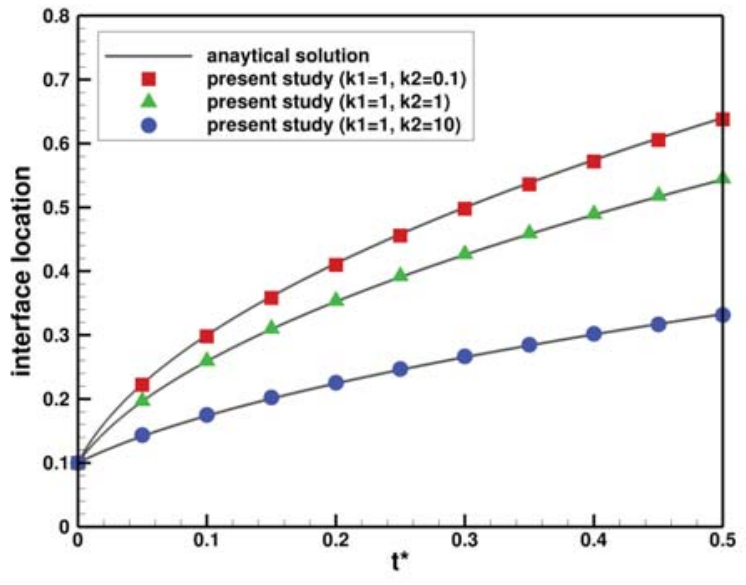

(a)

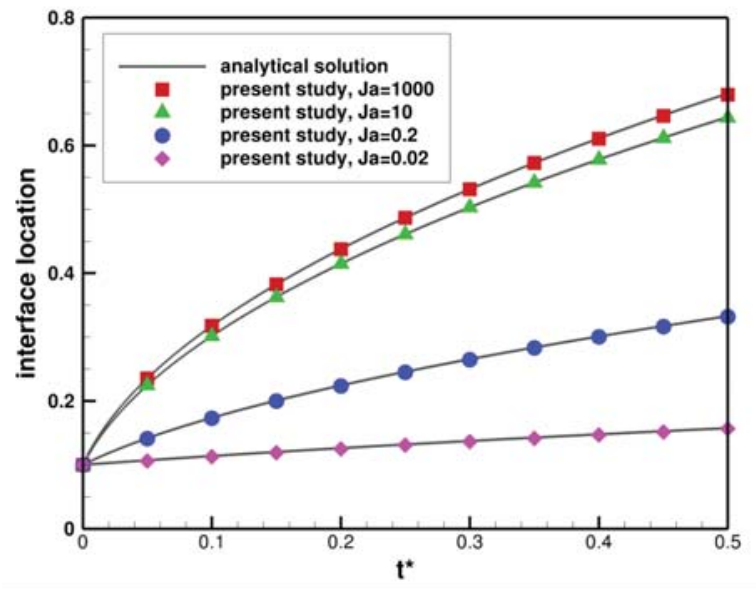

(b)

Figure 4: Comparison of the one-dimensional two-phases Stefan problem simulation with analytical solution at various Jacob numbers and material properties. (a) $\mathrm{Ja}=2$ with thermal conductivity of $0.1,1$, and 10 . Interface location matches very well with analytical solution for various heat transfer conditions. (b) For different Jacob number cases by changing latent heat and liquid-gas density ratio. The simulation results agree well with the analytical solution up to $\mathrm{Ja}=1000$.

\section{B. Droplet evaporation and fuel-vapor jet eruption by local droplet heating}

A model problem to represent flame-induced droplet evaporation and fuel jet eruption is set up as shown in Figure 5. A decane droplet is heated by a high temperature source representing a stationary flame at a constant temperature of $1500 \mathrm{~K}$. The configuration is axisymmetric around the centerline, with zero gravity and at the ambient pressure of $1 \mathrm{MPa}$, well below the critical pressure of $2.1 \mathrm{MPa}$ above which vapor and liquid phases become indistinguishable. The initial temperature of droplet and air is assumed $560 \mathrm{~K}$, which is $5.2 \mathrm{~K}$ lower than the saturation temperature of decane at the given pressure. The amount of the fuel evaporation is computed accurately on the decane vapor-to-liquid density ratio. Once evaporated, however, the fuel vapor is assumed to have the same density as the surrounding air for simplicity. Therefore, the fuel vapor is passively advected by the surrounding convection flow.

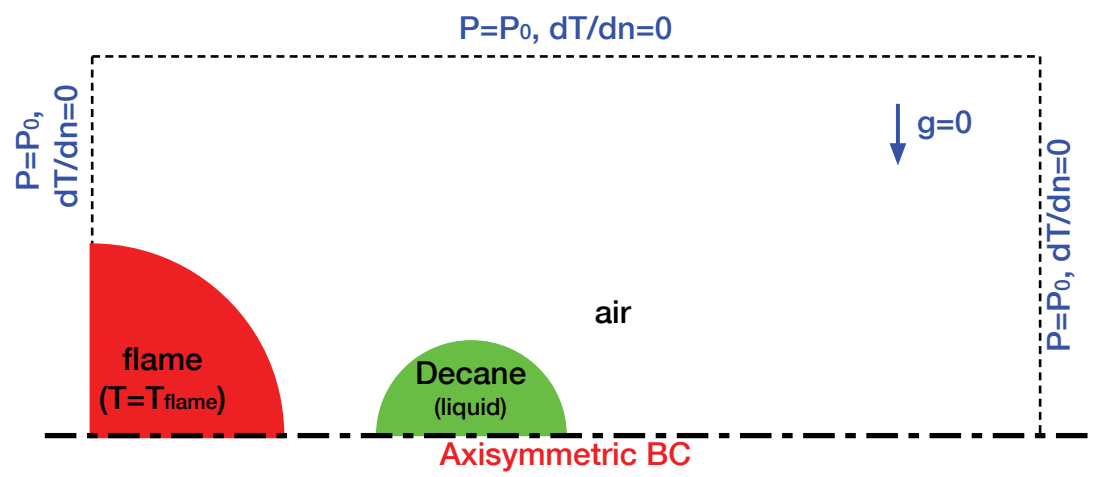

Figure 5: The illustration of the present droplet evaporation simulation in the axisymmetric computational domain. The stationary flame of constant temperature is located on the left side of a decane droplet in still air. Droplet diameter $d=1 \mathrm{~mm}$, spherical flame diameter $d_{\text {flame }}=2 d$, and the distance between droplet and flame $s=2 d$.

Figure 6 shows the temporal evolution of the evaporation rate and the aspect ratio. As heat is transferred from the flame of the left side to decane droplet, the evaporation begins at the left side, and the droplet size decreases. The evaporation rate increases while exhibiting a mild level of oscillation, then reaches a quasi-steady condition. The 


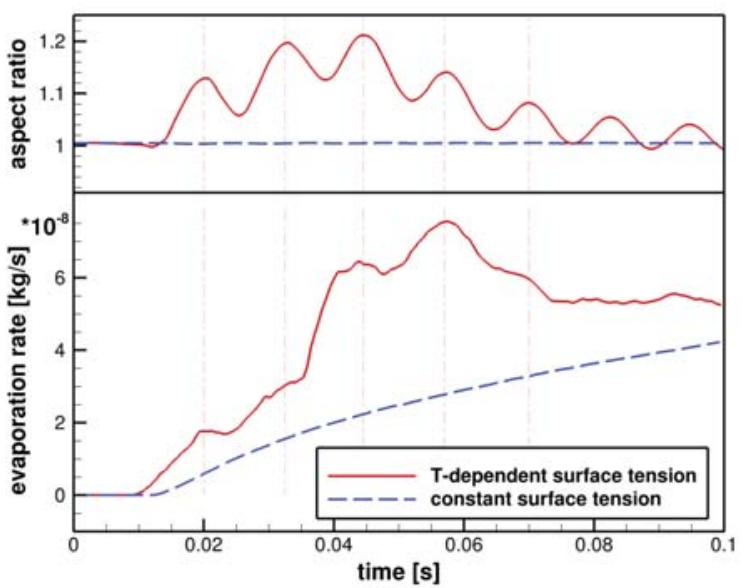

Figure 6: Temporal evolution of the droplet evaporation rate and the droplet aspect ratio. The droplet evaporation rate increases with a mild level of oscillation, and it is correlated with the oscillatory patterns of the droplet deformation.

oscillatory behavior is attributed to the fluctuations in the droplet aspect ratio shown in the same plot. In this study, the droplet is suddenly exposed to the high temperature source, causing an abrupt change in the surface tension gradient. Consequently, the droplet is deformed to a flatter shape. This in turn shortens the distance between the droplet and the heat source, yielding an increased evaporation rate. Upon recovery of the spherical shape by the surface tension, the evaporation rate also decreases. Figure 6 shows that the oscillatory patterns of the droplet deformation and the evaporation rate are correlated with each other. Therefore, although it is a physically correct result, the oscillatory droplet deformation is a result of the specific initial condition of abrupt initial heat-up.

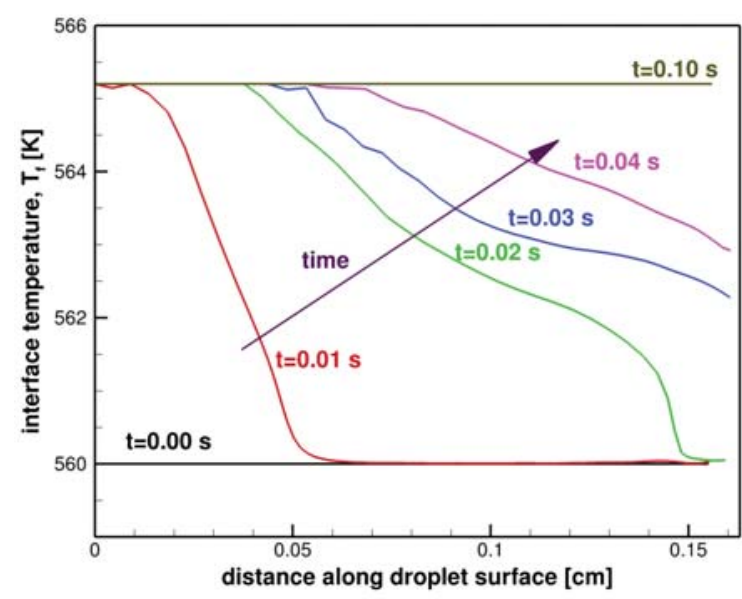

(a)

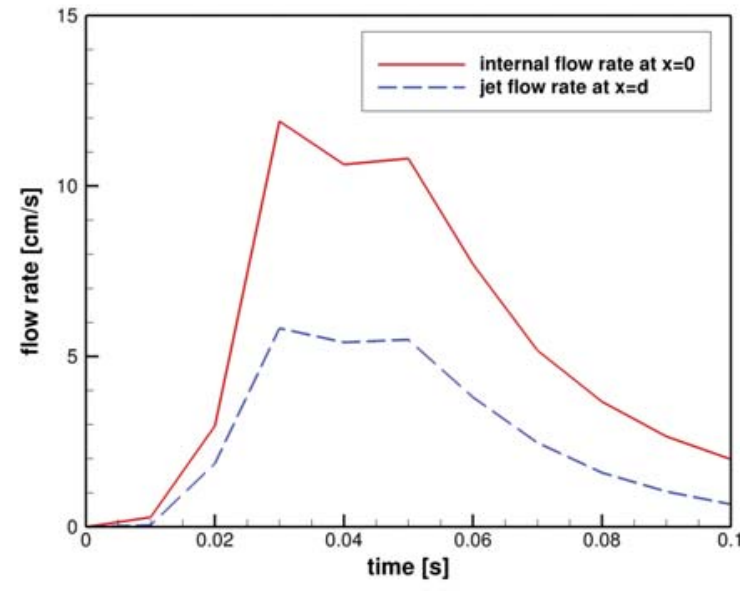

(b)

Figure 7: The history of the interface temperature distribution and flowrates by local droplet heating. (a) Interface temperature distribution along droplet surface. (b) The droplet internal flow rate and jet flow rate. Both of them show the same trend since they are induced by the same Marangoni effect due to the surface tension gradient along the droplet surface.

Figure 7(a) shows the change of interface temperature distribution along the droplet surface. Large initial temperature gradient is observed in the beginning, then the temperature gradient decreases as time goes. At $t=0.1 \mathrm{~s}$, the temperature gradient disapears completely as heat is transfered and the interface temperature reaches the boiling temperature on the whole droplet surface. These changes of the interface temperature gradient explain very well the changes of the resulting Marangoni effect in Figure 7(b). The droplet surface movement by Marangoni effect, which 


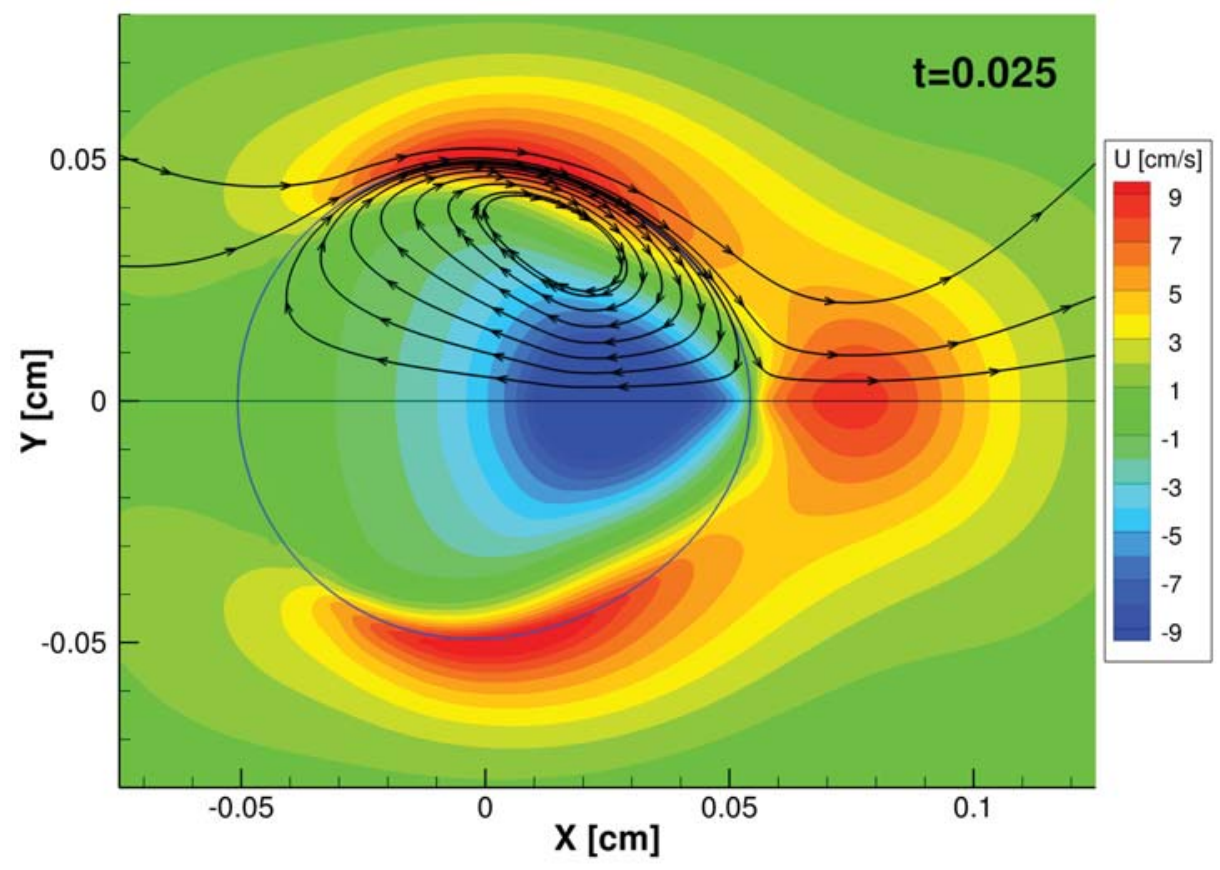

Figure 8: Streamlines of the shear flow around the droplet and inner Marangoni convection.

is induced by temperature-dependent surface tension gradient, causes inner flow circulation, namely Marangoni convection, and outer shear flow around the droplet surface. The droplet internal flow rate at the center of droplet is investigated in order to measure the strength of the inner Marangoni convection, and the jet flow rate is measured at $\mathrm{x}=\mathrm{d}$ along the centerline for the estimation of the shear flow. Both of them increase rapidly in the beginning with large temperature gradient, and decreases as temperature gradient disapears. The internal flow rate shows the same trend with jet flow rate since both of them are induced by the same surface tension gradient.

Figure 8 shows that local droplet heating creates a strong inner convection flow, referred to as the Marangoni convection. As heat is transferred, the Marangoni convection develops due to the surface tension gradient by temperature difference along droplet surface.

In order to confirm that the fuel vapor jet is induced by the temperature-dependent surface tension, another case was simulated with a constant surface tension model. Figure 9 shows the comparison of fuel-vapor concentration between two models. In the constant surface tension model of figure 9(a), the droplet is also evaporating as heat is transferred from the flame, but the fuel vapor is still on the flame side due to the lack of the induced shear flow. In contrast, the temperature-dependent surface tension model yields a significant fuel vapor jet eruption as shown in figure 9(b). Therefore, it is concluded that the surface tension variation is indeed a main cause of the experimentally observed internal flow and fuel vapor eruption. The fuel vapor jet promotes heat and mass transfer to the neighboring unburned droplets, and may provide an answer to the experimental observations ${ }^{8}$ at microgravity conditions that the evaporation increases with pressure until it reaches the critical condition.

Despite the results shown in figure 9, however, the magnitude of the fuel vapor jet eruption observed in the simulation is lower than that observed in the experiment. ${ }^{8}$ This is attributed to the differences in the simulation condition, in which the pressure was intentionally kept at $1 \mathrm{MPa}$ to stay away from near critical conditions. On the other hand, the experimental study reported strong jet flow near the critical pressure. As pressure increases, the latent heat become smaller and the decane droplet can be vaporized more easily. Furthermore, the actual flame is moves toward the droplet in the experiment, whereas in the present model the heat source is fixed in space. Nevertheless, the present study clearly demonstrates that the hypothesis of the surface tension-induced fuel vapor eruption is realistic at similar conditions observed in the experimental studies. More detailed high-fidelity models with multi-species gas-phase flow and extended thermodynamic properties up to near-critical pressure will be incorporated in future work. 


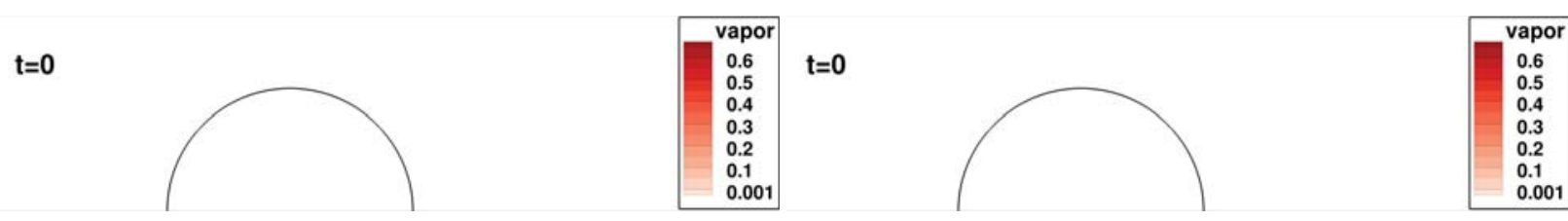

$t=0.01$

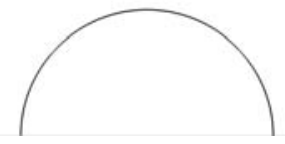

$t=0.02$

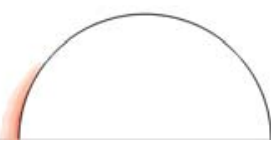

$t=0.03$

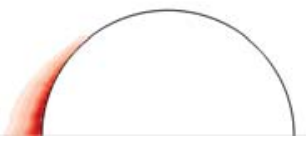

$t=0.04$

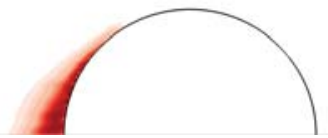

$t=0.06$

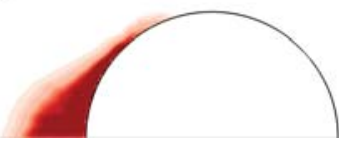

$t=0.08$

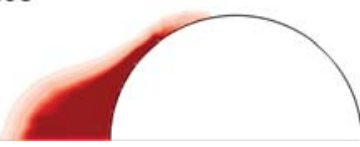

$t=0.1$

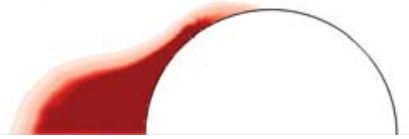

(a) $t=0.01$

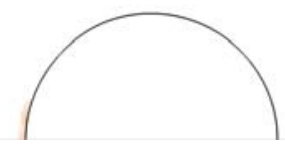

$t=0.02$

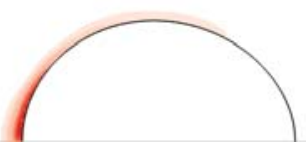

$t=0.03$

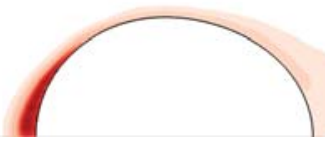

$t=0.04$

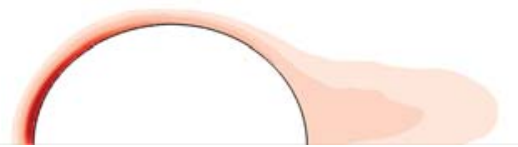

$t=0.06$

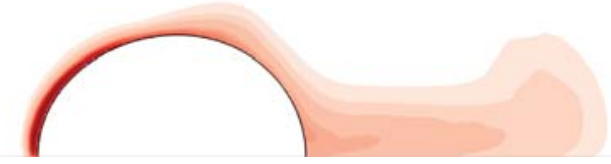

$t=0.08$

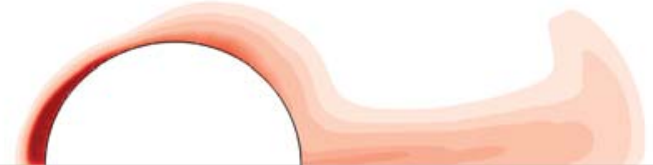

$t=0.1$

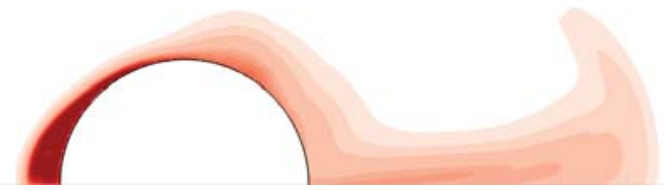

(b)

Figure 9: The parametric study with different surface tension models. (a) Constant surface tension model. The fuel vapor banks up on flame side. (b) Temperature-dependent surface tension model. The fuel vapor moves to wake region of droplet, and is erupted like jet. 


\section{Conclusions}

In the present study, the droplet evaporation by a localized heat source representing a neighboring flame was simulated as an attempt to explain the mechanism of the fuel vapor jet eruption observed experimentally at microgravity conditions. ${ }^{8}$ A modified Eulerian-Lagrangian method ${ }^{18}$ was implemented in order to account for the interfacial dynamics between liquid droplet and surrounding air with a new local phase change model. The accuracy of the developed model was validated with a test case Stefan problem. Two-dimensional axisymmetric model problem was subsequently conducted to represent conditions encountered in the experimental studies. It was found that the temperature difference by local droplet heating induces the corresponding surface tension gradient along the droplet surface, which develops shear flow around the droplet and strong internal Marangoni convection. The induced shear flow subsequently leads to a fuel vapor jet eruption by driving the evaporated fuel vapor to the wake region of the droplet. A parametric study with constant surface tension model confirmed that the mechanism of fuel vapor jet eruption was induced by the surface tension gradient resulting from the local heating.

\section{References}

${ }^{1}$ Law, C. K., "Recent Advances in Droplet Vaporization and Combustion," Progress in Energy and Combustion Science, Vol. 8, No. 3, 1982, pp. 171-201.

${ }^{2}$ Williams, F. A., Combustion Theory, Perseus Books, Reading, Massachusetts, 2nd ed., 1985.

${ }^{3}$ Godsave, G., "Studies of the Combustion of Drops in a Fuel Spray - the Burning of Single Drops of Fuel," Symposium (International) on Combustion, Vol. 4, No. 1, 1953, pp. 818-830.

${ }^{4}$ Kumagai, S. and Isoda, H., "Combustion of Fuel Droplets in a Falling Chamber," Symposium (International) on Combustion, Vol. 6, No. 1, 1957, pp. 726-731.

${ }^{5}$ Reichenbach, R., Squires, D., and Penner, S., "Flame Propagation in Liquid-Fuel Droplet Arrays," Symposium (International) on Combustion, Vol. 8, No. 1, 1961, pp. 1068-1073.

${ }^{6}$ Brzustowski, T., Sobiesiak, A., and Wojcicki, S., "Flame Propagation along an Array of Liquid Fuel Droplets at Zero Gravity," Symposium (International) on Combustion, Vol. 18, No. 1, 1981, pp. 265-273.

${ }^{7}$ Kato, S., Mizuno, H., Kobayashi, H., and Niioka, T., "Experiments on Flame Spread of a Fuel Droplet Array in a High-Pressure Ambience," JSME International Journal, Series B: Fluids and Thermal Engineering, Vol. 41, No. 2, 1998, pp. 322-330.

${ }^{8}$ Kobayashi, H., Park, J., Iwahashi, T., and Niioka, T., "Microgravity Experiments on Flame Spread of an n-decane Droplet Array in a High-Pressure Environment," Proceedings of the Combustion Institute, Vol. 29, No. 2, 2002, pp. 2603-2610.

${ }^{9}$ Kim, M., Chung, S., and Fujita, O., "Effect of AC Electric Fields on Flame Spread over Electrical Wire," Proceedings of the Combustion Institute, Vol. 33, No. 1, 2011, pp. 1145-1151.

${ }^{10}$ Prosperetti, A. and Tryggvason, G., Computational Methods for Multiphase Flow, Cambridge University Press, New York, 2007.

${ }^{11}$ Shyy, W. and Sim, J., "Computational Modeling for Multiphase Flows, Including Microgravity and Space Applications," Encyclopedia of Aerospace Engineering, edited by R. Blockley and W. Shyy, Vol. 1, chap. 51, Wiley, 2011, pp. 597-608.

${ }^{12}$ Uzgoren, E., Sim, J., and Shyy, W., "Marker-based, 3-D Adaptive Cartesian Grid Method for Multiphase Flow around Irregular Geometries," Communications in Computational Physics, Vol. 5, No. 1, 2009, pp. 1-41.

${ }^{13}$ Juric, D. and Tryggvason, G., "Computations of Boiling Flows," International Journal of Multiphase Flow, Vol. 24, No. 3, 1998, pp. 387410.

${ }^{14}$ Shin, S. and Juric, D., "Modeling Three-Dimensional Multiphase Flow Using a Level Contour Reconstruction Method for Front Tracking without Connectivity," Journal of Computational Physics, Vol. 180, No. 2, 2002, pp. 427-470.

${ }^{15}$ Son, G. and Dhir, V. K., "Numerical Simulation of Film Boiling Near Critical Pressures With a Level Set Method," Journal of Heat Transfer, Vol. 120, No. 1, 1998, pp. 183-192.

${ }^{16}$ Welch, S. W. J. and Wilson, J., "A Volume of Fluid Based Method for Fluid Flows with Phase Change," Journal of Computational Physics, Vol. 160, No. 2, 2000, pp. 662-682.

${ }^{17}$ Morgan, N. R., A New Liquid-Vapor Phase Transition Technique for the Level Set Method, Ph.D. thesis, Georgia Institute of Technology, Atlanta, 2005.

${ }^{18}$ Sim, J. and Shyy, W., "Interfacial Flow Computations Using Adaptive Eulerian-Lagrangian Method for Spacecraft Applications," International Journal for Numerical Methods in Fluids, Vol. 68, No. 11, 2012, pp. 1438-1456.

${ }^{19}$ Peskin, C. S., "The Immersed Boundary Method," Acta Numerica, Vol. 11, 2003, pp. 479-517. 